\title{
Are tag clouds useful for navigation? A network-theoretic analysis
}

Denis Helic*

Knowledge Management Institute, Graz University of Technology, Inffeldgasse 21a, 8010 Graz, Austria

E-mail: dhelic@tugraz.at

*Corresponding author

\section{Christoph Trattner}

Knowledge Management Institute, and Institute for Information Systems and Computer Media, Graz University of Technology, Inffeldgasse 16c, A-8010 Graz, Austria

E-mail: ctrattner@iicm.edu

\section{Markus Strohmaier}

Knowledge Management Institute,

and

Know-Center,

Graz University of Technology,

Inffeldgasse 21a, 8010 Graz, Austria

E-mail: markus.strohmaier@tugraz.at

\section{Keith Andrews}

Institute for Information Systems and Computer Media, Graz University of Technology, Inffeldgasse 16c, $8010 \mathrm{Graz}$, Austria E-mail: kandrewsg@iicm.edu

\begin{abstract}
It is a widely held belief among designers of social tagging systems that tag clouds represent a useful tool for navigation. This is evident in the increasing number of tagging systems offering tag clouds, which hints towards an implicit assumption that tag clouds support efficient navigation. In this paper, we test this assumption from a network-theoretic perspective, and show that in many cases, it does not hold. We first model navigation in tagging systems and then simulate the navigation process in such a graph. We analyse the navigability of three tagging datasets with regard to different user interface restrictions imposed by tag clouds. Our results confirm that tag clouds have efficient navigation properties in theory, but they also show that popular user interface decisions, such as 'pagination' significantly impair their navigability. Finally, we identify a number of avenues for further research and the design of novel tag cloud construction algorithms.
\end{abstract}


Keywords: tagging; tag clouds; navigability; simulation; user interface; pagination.

Reference to this paper should be made as follows: Helic, D., Trattner, C., Strohmaier, M. and Andrews, K. (2011) 'Are tag clouds useful for navigation? A network-theoretic analysis', Int. J. Social Computing and Cyber-Physical Systems, Vol. 1, No. 1, pp.33-55.

Biographical notes: Denis Helic is an Associate Professor at the Institute for Knowledge Management at Graz University of Technology, Austria. $\mathrm{He}$ received his MSc (Dipl. Ing.) in Computer Technics, $\mathrm{PhD}$ in Computer Science (with honours) from Graz University of Technology, and postdoctoral lecture thesis for the field applied computer science at the Graz University of Technology. His research interests include multimedia and hypermedia information systems, the web, semantic modelling on the web, and e-learning systems. He published more than 50 research papers in refereed international journals and conferences and won a number of conference best-paper awards related to his e-learning research work.

Christoph Trattner is a Scientific Research Assistant and $\mathrm{PhD}$ candidate at Knowledge Management Institute and Institute for Information Systems and Computer Media at Graz University of Technology, Austria. He completed his MSc with honours in March 2009 and BSc in Telematics at Graz University of Technology, Austria in March 2008. His research field is social computing. The focus of his $\mathrm{PhD}$ research is on the navigability of social tagging systems. He is funded by - BMVIT - the Federal Ministry for Transport, Innovation and Technology, Programme Line 'Forschung, Innovation und Technologie für Informationstechnologie', project 'NAVTAG - Improving the navigability of tagging systems'.

Markus Strohmaier is an Assistant Professor at the Knowledge Management Institute at Graz University of Technology, Austria since June 2007. From March 2006 to May 2007, he was a Postdoctoral Research Fellow at the Department of Computer Science at University of Toronto, Canada funded by an FWF Erwin Schroedinger Postdoctoral Fellowship. He was the Principal Investigator (PI) of a three-year FWF Research Grant (TransAgere) that focuses on the role of (human) agents and goals on the social web (2007-2010).

Keith Andrews is a Tenured Associate Professor at the Institute for Information Systems and Computer Media (IICM) at Graz University of Technology, in Austria. Having done work in the fields of computer graphics, 3D worlds, hypermedia, and the web, he is currently pursuing his research in the fields of information visualisation and usability. He was a Programme co-Chair of the IEEE Symposium on Information Visualisation in 2001 and 2002, and General Chair in 2005.

\section{Introduction}

In social tagging systems such as Flickr and Delicious, tag clouds have emerged as an interesting alternative to traditional forms of navigation and hypertext browsing. The basic idea is that tag clouds provide navigational clues by aggregating tags and corresponding resources from multiple sources, and by displaying them in a visually appealing fashion. Users are presented with these tag clouds as a means for exploring and navigating the resource space in social tagging systems. 
While tag clouds can potentially serve different purposes, there seems to be an implicit assumption among engineers of social tagging systems that tag clouds are specifically useful to support navigation. This is evident in the large-scale adoption of tag clouds for interlinking resources in numerous systems such as Flickr, Delicious, and BibSonomy. However, this navigability assumption has hardly been critically reflected [with some notable exceptions, e.g., Hearst and Rosner (2008)], and has largely remained untested in the past. In this paper, we will demonstrate that the prevalent approach to tag cloud-based navigation in social tagging systems is highly problematic with regard to network-theoretic measures of navigability. In a series of experiments, we will show that the navigability assumption only holds in very specific settings, and for the most common scenarios, we can assert that it is wrong.

While recent research has studied navigation in social tagging systems from user interface (Mesnage and Carman, 2009; Rivadeneira et al., 2007; Sinclair and Cardew-Hall, 2008) and network-theoretic (Neubauer and Obermayer, 2009) perspectives, the unique focus of this paper is the intersection of these issues. With that focus, we want to answer questions such as: how do user interface constraints of tag clouds affect the navigability of tagging systems? And how efficient is navigation via tag clouds from a network-theoretic perspective?

Particularly, we will first:

1 investigate the intrinsic navigability of tagging datasets without considering user interface effects

2 and then take pragmatic user interface constraints into account

3 next, we will demonstrate that for many social tagging systems, the navigability assumption does not hold

4 and then we will use our findings to illuminate a path towards improving the navigability of tag clouds

5 thereafter, we will argue that any new tag-cloud construction algorithm will need to balance the trade-off between navigational and semantic penalties induced by the network generation process

6 and finally, we will present a simple method for estimating the semantic penalty.

To the best of our knowledge, this paper is among the first to study what we have called the navigability assumption of tag clouds, i.e., the widely held belief that tag clouds are useful for navigating social tagging systems. One of the main results of this paper is a more critical stance towards the usefulness of tag clouds as a navigational aid in tagging systems. We argue that in order to make use of the full potential of tag clouds, new ways of thinking about tag cloud algorithms are needed.

The paper is structured as follows: in Section 2, we present our network-theoretic approach to assessing navigability of tagging systems. Section 3 describes the analysed datasets. Section 4 presents and discusses the analysis results. Based on our findings, we call for and discuss new ideas for tag cloud algorithms in Section 5. In Section 6, we sketch a new algorithm for constructing tag clouds and present a method for estimating the semantic properties of the network generated by that algorithm. Section 7 provides an overview of related work. Finally, Section 8 concludes the paper and presents directions for future work. 


\section{Network-theoretic model of navigation in tagging systems}

A tagging dataset is typically modelled as a tripartite hypergraph with $V=R \cup U \cup T$, where $R$ is the resource set, $U$ is the user set, and $T$ is the tag set (Cattuto et al., 2007; Schmitz et al., 2006; Ramezani et al., 2009). An annotation of a particular resource with a particular tag produced by a particular user is a hyperedge $(r, t, u)$, connecting three nodes from these three disjoint sets.

Such a tripartite hypergraph can be mapped onto three bipartite graphs connecting users and resources, users and tags, and tags and resources. For different purposes, it is often more practical to analyse one or more of these bipartite graphs. For example, in the context of ontology learning, the bipartite graph of users and tags has been shown to be an effective projection (Mika, 2007).

In this paper, we focus on tag-resource bipartite graphs. These graphs naturally reflect the way users are supposed to adopt tag clouds for navigating social tagging systems. For example, in many tagging systems, tag clouds are intended to be used in the following way:

1 the system presents a tag cloud to the user

2 the user selects a tag from the tag cloud

3 the system presents a list of resources tagged with the selected tag

4 the user selects a resource from the list of resources.

5 the system transfers the user to the selected resource, and the process potentially starts anew.

We will study this general interaction schema and model it with a simulated user moving along the edges of the tag-resource bipartite graph and alternately visiting tag and resource nodes.

To that end, we introduce a network-theoretic approach for assessing the navigability and the efficiency of navigability in such a bipartite graph. Ever since Milgram's (1967) small world experiment, researchers aimed to understand 'navigability' and in particular 'efficient' navigation of networks (for details see Section 7). Among others, two important results stem from this line of research:

1 there exist short paths between people (nodes) in a social network

2 people are able to navigate 'efficiently' through the network having only local knowledge of the network, i.e., knowing only their personal contacts.

Kleinberg (2000a, 2000b, 2001) and also independently Watts et al. (2002) formalised these properties concluding that a navigable network has a short path between all - or almost all - nodes in the network (Kleinberg, 2001). Formally, such a network has a low diameter bounded polylogarithmically, i.e., by a polynomial in $\log N$, where $N$ is the number of nodes in the network, and there exists a giant component, i.e., a strongly connected component containing almost all nodes (Kleinberg, 2001). Additionally, an 'efficiently' navigable network possesses certain structural properties so that it is possible to design efficient decentralised search algorithms (algorithms that only have local knowledge of the network) (Kleinberg, 2000a, 2000b, 2001). The delivery time (the 
expected number of steps to reach an arbitrary target node) of such algorithms is polylogarithmic or at most sub-linear in $N$.

User navigation in hypertext systems is naturally modelled as a decentralised search, i.e., at each particular node in the network, users select a new node having only local knowledge of the network and following the idea that the selected node would bring them closest to their destination node. We use this model to investigate the navigability of tag clouds next.

\section{Experimental setup}

In the following, we conduct experiments aiming to shed light on the navigability of tag clouds in social tagging systems. We are particularly interested in studying how design decisions, such as what tags to include in a tag cloud or how many tags to display, effect the navigability of tag clouds. While, today, designers often base such decisions on intuition or heuristics, it is our goal to study the consequences of these decisions experimentally, i.e., by exploring their empirical effects on the network.

In our experiments, we used three datasets covering a range of different settings.

- Dataset Austria-Forum: This dataset consists of annotations from an Austrian encyclopaedia called Austria-Forum. ${ }^{1}$ The dataset contains 32,245 annotations and 12,837 unique resources. The system is at an early phase of adoption, i.e., not many users currently contribute new tags.

- Dataset BibSonomy: This dataset ${ }^{2}$ contains nearly all 916,495 annotations and 235,339 unique resources from a dump of BibSonomy (Hotho et al., 2006) until 2009-01-01. Annotations from known spammers have been excluded from the dataset. This dataset is obtained from a more mature tagging system.

- Dataset CiteULike: This dataset contains 6,328,021 annotations and 1,697,365 unique resources and is available online. ${ }^{3}$ Again, this is a dataset acquired from a more mature tagging system.

Dataset Austria-Forum represents a tagging system at an early stage of adoption. Datasets BibSonomy and CiteULike are tagging systems which have reached a certain level of maturity (i.e., attracted a larger set of active users). While all three systems adopt tag clouds for navigational purposes, their specific approaches vary. However, because the datasets contain complete information about the tripartite graph, we can experimentally manipulate the data in a way that simulates different approaches to tag cloud construction consistently across all datasets. We will describe how we manipulate the data to simulate different user interface constraints next.

\subsection{User interface issues}

The first user interface restriction which we model is the size of a tag cloud, i.e., the maximal number of tags displayed in a tag cloud. While different tagging systems implement different design choices, we can simulate alternative choices across all datasets. For example, in some tagging systems the maximum number of tags in a tag cloud might be 20, while in others it might be much larger. 
Another important issue of tag clouds is the algorithm used to select the tags to display in a tag cloud. While, in theory, there are many ways to compute and visualise tag clouds (Eda et al., 2009; Kaser and Lemire, 2007; Rivadeneira et al., 2007), in practice, many tagging systems follow a simple resource-specific, TopN algorithm. In resource-specific approaches to tag cloud construction, only tags assigned to the corresponding resources are considered. In TopN approaches, the top $n$ tags with the highest resource-specific frequency are chosen for display in the corresponding tag cloud. In cases where less than $n$ tags per resource are available, the remaining slots are left empty.

For the experiments aiming to study the navigational assumption, we used the TopN algorithm (because it is the most common) to reconstruct simulated networks of resource-specific tag clouds for our three datasets.

Popular tags in a mature tagging system can cover hundreds or even thousands of resources, which exceeds the pragmatic limits of a system's user interface. In this situation, tagging systems usually resort to limiting the set of resources being displayed for a given tag (e.g., by sorting and 'paginating' the list of corresponding resources). To model such limits, we introduce a pragmatic parameter, the length of the resource list being presented, and denote it henceforth with $k$.

In the majority of tagging systems, the resource lists presented after selecting a tag are usually sorted reverse-chronologically (the resources most recently tagged are listed first). For simplicity, in our experiments, we select the $k$ resources for $k$-limited resource lists randomly.

\section{Results}

\subsection{Intrinsic navigability of tagging systems}

We start our study by analysing the navigability of tagging systems in a synthetic network-theoretic case, i.e., without taking any user interface restrictions into account. The first row in each of Tables 1(a), 1(b), and 1(c) present the obtained results. The results show the existence of a giant component connecting almost all of the nodes $(98 \%)$, as well as the existence of a low effective diameter (less than 7, i.e., it is less than polynomial in $\log N$, see Figure 1).

The only exception here is the Austria-Forum dataset. We speculate that the reason for that is due to the system being in an early adoption stage. While the effective diameter of the Austria-Forum dataset is larger than the one in the two other datasets (see Figure 1), it is still limited polylogarithmically, whereas the giant component contains only $77 \%$ of nodes. This result suggests that the navigability assumption depends on the adoption stage of the tagging system under investigation, i.e., the assumption may only hold for more mature tagging systems BibSonomy or CiteULike. We leave the issue of identifying the point in time where immature tagging systems transition to tagging systems exhibiting more useful navigational properties to future research. At this point, we simply observe that the navigation assumption is sensitive to the stage of adoption of a tagging system. 
Table 1 Navigational properties of the Austria-Forum, BibSonomy, and CiteULike tagging systems

\begin{tabular}{|c|c|c|c|c|}
\hline \multicolumn{5}{|c|}{ (a) Austria-Forum } \\
\hline$U I R$ & $G C$ & $E D$ & $U I A$ & $N A D T$ \\
\hline None & 0.77 & 10.73 & None & Sub-lin. \\
\hline$n=5$ & 0.75 & 10.99 & TopN & Sub-lin. \\
\hline$n=10$ & 0.76 & 11.3 & TopN & Sub-lin. \\
\hline$n=20$ & 0.76 & 11.97 & TopN & Sub-lin. \\
\hline$n=30$ & 0.76 & 11.05 & TopN & Sub-lin. \\
\hline$k=5$ & 0.36 & 12.04 & Chron. & Unnav. \\
\hline$k=10$ & 0.47 & 11.16 & Chron. & Unnav. \\
\hline$k=20$ & 0.56 & 10.31 & Chron. & Unnav. \\
\hline$k=30$ & 0.6 & 10.68 & Chron. & Unnav. \\
\hline \multicolumn{5}{|c|}{ (b) BibSonomy } \\
\hline$U I R$ & $G C$ & $E D$ & $U I A$ & $N A D T$ \\
\hline None & 0.98 & 6.96 & None & Sub-lin. \\
\hline$n=5$ & 0.94 & 6.8 & TopN & Sub-lin. \\
\hline$n=10$ & 0.97 & 6.87 & TopN & Sub-lin. \\
\hline$n=20$ & 0.98 & 6.84 & TopN & Sub-lin. \\
\hline$n=30$ & 0.98 & 6.91 & TopN & Sub-lin. \\
\hline$k=5$ & 0.31 & 6.82 & Chron. & Unnav. \\
\hline$k=10$ & 0.4 & 6.62 & Chron. & Unnav. \\
\hline$k=20$ & 0.5 & 6.61 & Chron. & Unnav. \\
\hline$k=30$ & 0.54 & 6.65 & Chron. & Unnav. \\
\hline \multicolumn{5}{|c|}{ (c) CiteULike } \\
\hline$U I R$ & $G C$ & $E D$ & $U I A$ & $N A D T$ \\
\hline None & 0.98 & 6.85 & None & Sub-lin. \\
\hline$n=5$ & 0.93 & 6.97 & TopN & Sub-lin. \\
\hline$n=10$ & 0.95 & 7.07 & TopN & Sub-lin. \\
\hline$n=20$ & 0.97 & 7.17 & TopN & Sub-lin. \\
\hline$n=30$ & 0.97 & 6.98 & TopN & Sub-lin. \\
\hline$k=5$ & 0.27 & 6.89 & Chron. & Unnav. \\
\hline$k=10$ & 0.36 & 6.95 & Chron. & Unnav. \\
\hline$k=20$ & 0.44 & 6.91 & Chron. & Unnav. \\
\hline$k=30$ & 0.48 & 7.05 & Chron. & Unnav. \\
\hline
\end{tabular}

Notes: UIR = UI restriction, $\mathrm{GC}=$ giant component, $\mathrm{ED}=$ effective diameter, UIA = UI algorithm, NADT = navigation algorithm delivery time, Chron. $=$ chronological algorithm, Sub-lin. = sub-linear, Unnav. = unnavigable network. 
Figure 1 Hop plots for three different tagging datasets (see online version for colours)

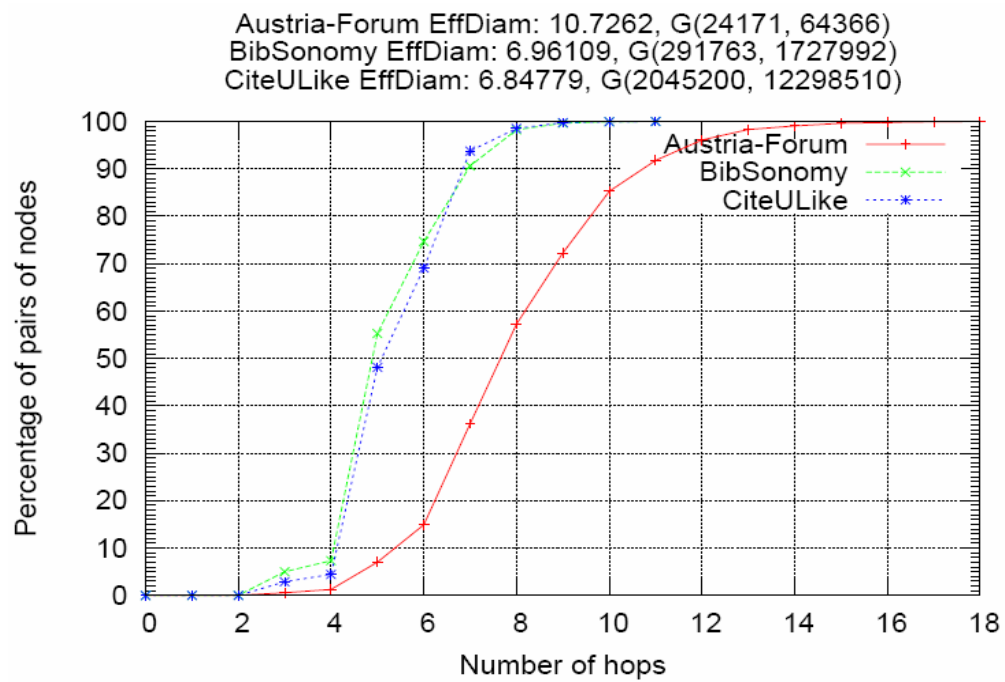

Notes: We can observe the shrinking diameter phenomenon. The two mature datasets (Bibsonomy and CiteULike, the two lines on the left) exhibit a small diameter, while the Austria-Forum (a tagging system in an early adoption phase, the line on the right) exhibits a larger diameter, and a larger ratio of long distances between nodes.

Source: Leskovec et al. (2005)

Result 1 The usefulness of tag clouds for navigation is sensitive to the phase of adoption of the social tagging system.

Figures 2(a), 2(b), and 2(c) show tag (blue), resource (green), and degree (red) distributions for the analysed datasets. The tag and resource distributions were obtained by analysing a unidirectional bipartite graph, i.e., a graph with only directed links from tags to resources. The out-degree distribution and the in-degree distribution in this graph correspond to tag distribution and to resource distribution respectively. For certain ranges of degrees, both distributions are power law distributions. There are deviations in the tail of the tag distribution - these stem from the system tags assigned to imported resources [see Figures 2(b) and 2(c)]. The vertical line in the tail of Figure 2(c) comes from the existence of synonym tags in the dataset. The resource distributions exhibit an exponential cut-off in the tail [see Figure 2(b)], a deviation in the tail stemming from a test resource [see Figure 2(a)], and a power law distribution as in Figure 2(c).

The degree distribution of the undirected bipartite graph [the red line in Figures 2(a), 2(b) and 2(c)] combines both tag and resource distributions. For lower degrees, the combined degree distribution takes the form of the resource distribution, i.e., the number of resources with low frequencies dominates the number of tags with low frequencies. For higher degrees, the combined distribution takes the form of the tag distribution, i.e., there are more tags with high frequencies than resources with high frequencies. The tag distribution is two or more orders of magnitude larger than the resource distribution, i.e., the tag distribution strongly dominate the resource distribution for higher degrees. That means that the network hubs (high-degree nodes) are the 'head' tags, i.e., the top tags for TopN tag cloud construction algorithms. 
Figure 2 Tag, resource, and degree distributions for the three datasets, (a) Austria-Forum (b) BibSonomy (c) CiteULike (see online version for colours)

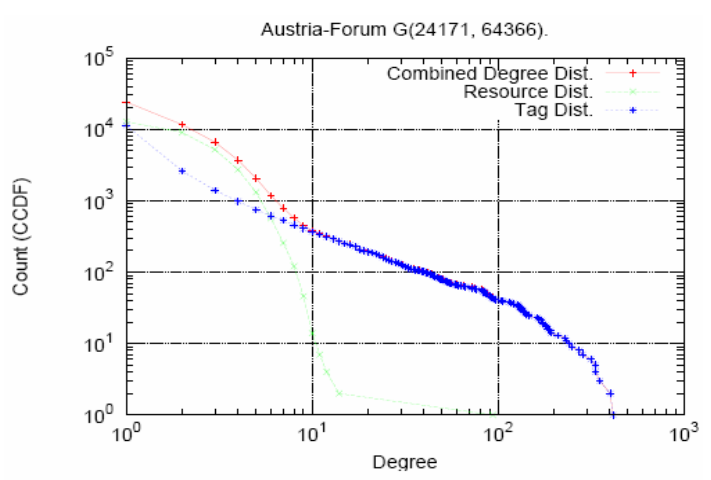

(a)

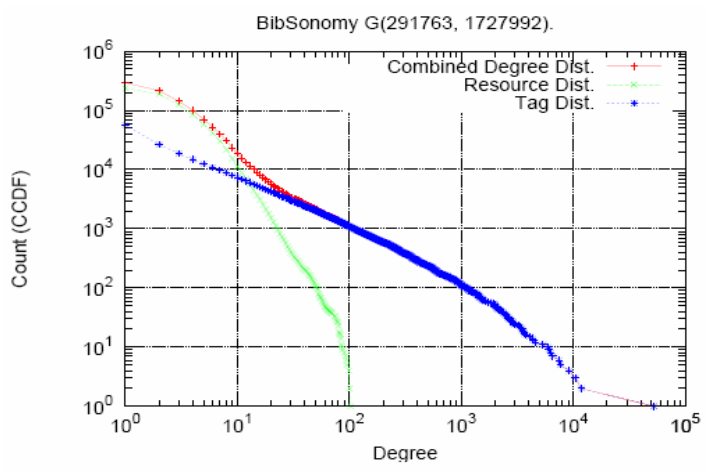

(b)

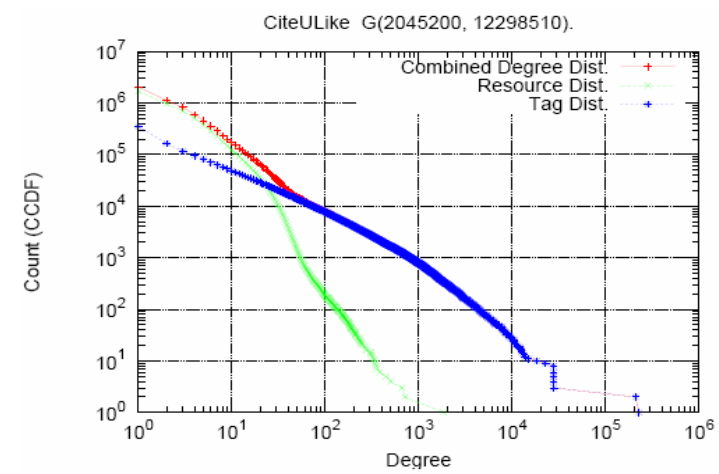

(c)

Notes: We can observe that the tag degrees are two or more orders of magnitude greater than the resource degrees, i.e., the tag distribution strongly dominates the resource distribution for higher degrees. Therefore, the network hubs (high-degree nodes) are the 'head' tags - the top tags for TopN tag cloud construction algorithms. It is therefore to expect that limiting of the tag cloud size will not influence the navigability of the tag-resource network as the hub nodes are still present in the network. 
Due to the existence of a giant component and a low diameter, tagging systems are intrinsically navigable. In Adamic et al. (2001) show the existence of efficient decentralised navigation and search algorithms for power law networks. In principle, a user could first navigate to a hub (which is typically achieved in a few hops in a power law network) and since hubs have a large out-degree, one can reach the destination node easily. The delivery time of the algorithm is sub-linear, although the number of inspected nodes in the worst-case is $O(N)$, since sometimes the user needs to inspect all outgoing links from a hub.

Result 2 Tagging networks are navigable power-law networks. For power law networks, efficient sub-linear decentralised navigation algorithms exist.

\subsection{Tag cloud size}

Rows two to five of Tables 1(a), 1(b), and 1(c) show the results of applying the TopN algorithm to limit the tag cloud size on the analysed datasets. From a network-theoretic point of view, limiting the tag cloud size means limiting the out-degree of the resource nodes in the bipartite graph. The out-degree of the resource nodes is two orders of magnitude smaller then the out-degree of the tag nodes, indicating there are no resource 'hubs' in the network. Therefore, limiting the tag cloud size does not influence the network to a large extent. In other words, the structure of the network is still maintained, i.e., the network remains a navigable network with navigation efficiency inherent to power law networks.

Result 3 Limiting the tag cloud size to practically feasible sizes (e.g., 5, 10, or more) does not influence navigability.

\subsection{Pagination}

Rows six to nine of Tables 1(a), 1(b), and 1(c) contain the results of simulating pagination with resource lists sorted reverse-chronologically. Even without experiments, it is evident that limiting the number of links going out from a tag node has destructive effects on the resulting network. In other words, limiting the out-degree of hub nodes in a power-law network destroys the connectivity of the network as a whole. Our experiments show exactly that: the giant component collapses, and the largest strongly connected component now only contains around $50 \%$ or less nodes. As such, pagination destroys network navigability, and the navigability assumption only holds when we assume that users would be able and willing to inspect long lists $(>10.000)$ of resources per tag, which is not reasonable. For example, we know from search query log research that users rarely click on links beyond the first result page (Zhang et al., 2009). This yields our final result:

Result 4 Limiting the out-degree of high frequency tags (e.g., through pagination with resource lists sorted reverse-chronologically) leaves the network vulnerable to fragmentation. This destroys navigability of prevalent approaches to tag clouds. 


\section{Implications}

The previous analysis illustrated the vulnerability of tagging networks to the pagination effect, where a limit is placed on the number of links going out from paginated tags, i.e., tags with frequency higher than the pagination parameter $k$. This vulnerability is mainly due to the simplicity of the common pagination algorithm, i.e., the resource list is simply sorted reverse-chronologically and only the $k$ most recently tagged resources are presented to the user. The algorithm does not take into account the current user context, i.e., the resource where the user clicks on a paginated tag. Rather the same reverse-chronologically resource list is presented for a given paginated tag throughout the system.

Let us now investigate possibilities to recover the navigability of tagging networks by means of alternative tag construction algorithms. To this end, we introduce an adapted pagination algorithm. A simple generalisation of the pagination algorithm is to select $k$ different resources out of all resources tagged with a given paginated tag, depending on the current user context, i.e., depending on the resource where the user activates a paginated tag. Let us denote the resources list of a given paginated tag $t$ with $R_{t}$. In this case, a particular selection of resources for $t$ becomes a function of a given resource and parameter $k$, i.e., $L_{t}=f(r, k)$. In other words, each paginated tag is replaced by as many resource-specific tags $\left(t_{r}\right)$ as there are resources in its resource list. Each resource-specific tag is then connected to resources computed by $f(r, k)$. The pseudo-code of the generalised algorithm is given in Figure 3.

We now discuss some potential functions $f(r, k)$ for selecting resources from the available resource pool and analyse their influence on network navigability.

Figure 3 Generalised pagination algorithm

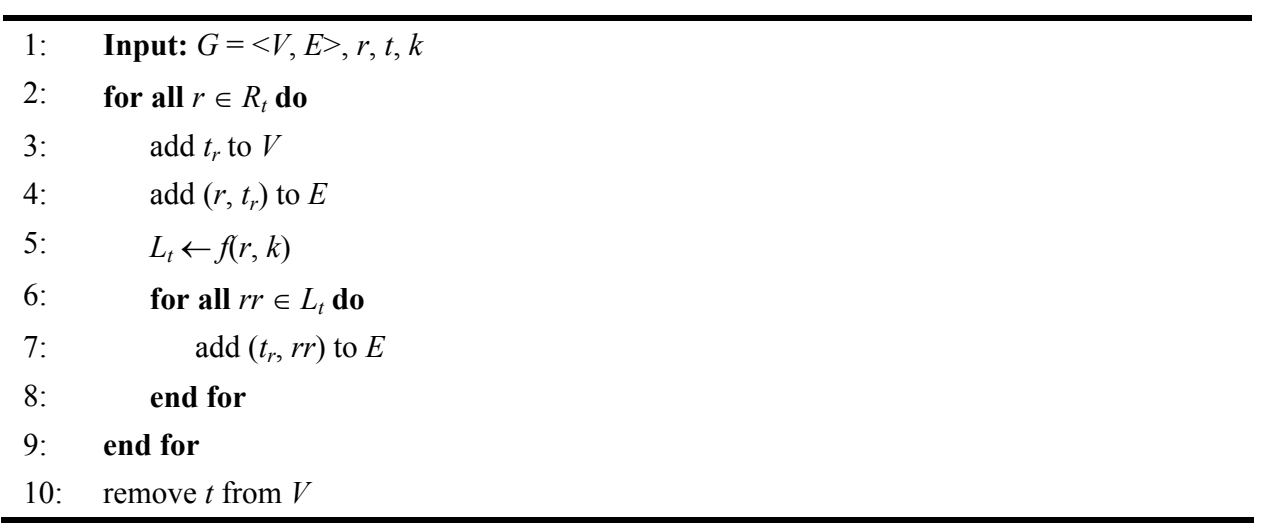

\subsection{Random link selection}

A first obvious choice for $f(r, k)$ is to select $k$ resources uniformly at random. This approach generates a random graph as introduced by Erdos and Renyi (1960) for each given paginated tag. As Bollobás and de la Vega (1982), and Bollobás and Chung (1988) showed, graphs generated uniformly at random are typically connected and have - with a high probability - a diameter bound by $\log N$ (already for out-degrees $k \geq 3$ ). However, since there are no structural clues in a randomly generated network, a decentralised 
search algorithm will need to inspect, in the worst case, all nodes of the network in order to reach a destination node from the given starting node.

Table 2 shows the results of a random pagination algorithm on the three test datasets. All three networks become strongly connected with a giant component even for low values of $k$. As expected, all three networks also possess a low diameter.

Table 2 Navigational properties of the Austria-Forum, BibSonomy, and CiteULike tagging systems with a random pagination algorithm

\begin{tabular}{lccll}
\hline \multicolumn{5}{c}{ (a) Austria-Forum } \\
\hline UIR & $G C$ & $E D$ & UIA & NADT \\
\hline$k=5$ & 0.86 & 11.7 & Random & Linear \\
$k=10$ & 0.86 & 11.02 & Random & Linear \\
$k=20$ & 0.85 & 10 & Random & Linear \\
$k=30$ & 0.84 & 10.42 & Random & Linear \\
\hline & & $($ b) BibSonomy & & \\
\hline$U I R$ & $G C$ & ED & UIA & NADT \\
\hline$k=5$ & 0.99 & 8.75 & Random & Linear \\
$k=10$ & 0.99 & 6.97 & Random & Linear \\
$k=20$ & 0.99 & 6.75 & Random & Linear \\
$k=30$ & 0.99 & 6.46 & Random & Linear \\
\hline & & $(c)$ CiteULike & & \\
\hline$U I R$ & $G C$ & ED & UIA & NADT \\
\hline$k=5$ & 0.99 & 7.98 & Random & Linear \\
$k=10$ & 0.99 & 7.88 & Random & Linear \\
$k=20$ & 0.99 & 7.13 & Random & Linear \\
$k=30$ & 0.99 & 6.86 & Random & Linear \\
\hline
\end{tabular}

Notes: UIR $=$ UI restriction, $\mathrm{GC}=$ giant component, $\mathrm{ED}=$ effective diameter,

$\mathrm{UIA}=\mathrm{UI}$ algorithm, NADT $=$ navigation algorithm delivery time.

\subsection{Hierarchical network model}

In Kleinberg (2001), he introduced the hierarchical network model and elegantly proved that it is possible to design efficient decentralised search algorithms for such networks with a delivery time polynomial in $\log N$ (for details see Section 7). Put simply, Kleinberg showed that, if the nodes of a network can be organised into a hierarchy, then such a hierarchy provides a probability distribution for connecting the nodes in the network. The resulting network is efficiently navigable. A special case of the hierarchical network model is given when there is a constant number of links leaving a node, i.e., when the out-degree of a node is limited by a parameter $k$ as it is the case with pagination. In this case, the tree leaves contain so-called clusters of nodes, i.e., a collection of a certain constant number of nodes.

Thus, we developed a hierarchical network generator that: 
1 sorts the resource list of a given paginated tag by frequency

2 creates resource clusters of size ten by traversing the sorted resource list sequentially

3 creates a balanced $b$-ary $(b=5)$ tree where the number of leaves is equal to the number of the resource clusters

4 traverses the tree in post-order from left to right and attaches resource clusters to the tree leaves

5 uses this tree structure to obtain the link probability distribution for connecting a resource-specific tag node with resources of a given paginated tag.

Table 3 Navigational properties of the Austria-Forum, BibSonomy, and CiteULike tagging systems with a hierarchical pagination algorithm

\begin{tabular}{lllll}
\hline \multicolumn{5}{c}{ (a) Austria-Forum } \\
\hline UIR & $G C$ & $E D$ & UIA & NADT \\
\hline$k=5$ & 0.85 & 12.03 & Hier. & Polylog. \\
$k=10$ & 0.86 & 10.62 & Hier. & Polylog. \\
$k=20$ & 0.85 & 9.29 & Hier. & Polylog. \\
$k=30$ & 0.84 & 9.71 & Hier. & Polylog. \\
\hline & & $(b)$ BibSonomy & & \\
\hline UIR & $G C$ & ED & UIA & NADT \\
\hline$k=5$ & 0.99 & 8.82 & Hier. & Polylog. \\
$k=10$ & 0.99 & 7.62 & Hier. & Polylog. \\
$k=20$ & 0.99 & 6.94 & Hier. & Polylog. \\
$k=30$ & 0.99 & 6.75 & Hier. & Polylog. \\
\hline & & $(c)$ CiteULike & & \\
\hline$U I R$ & $G C$ & ED & UIA & NADT \\
\hline$k=5$ & 0.99 & 8.76 & Hier. & Polylog. \\
$k=10$ & 0.99 & 7.6 & Hier. & Polylog. \\
$k=20$ & 0.99 & 6.36 & Hier. & Polylog. \\
$k=30$ & 0.99 & 5.89 & Hier. & Polylog. \\
\hline
\end{tabular}

Notes: UIR = UI Restriction, GC = Giant Comp., ED = Eff. Diameter, UIA = UI

Algorithm, NADT = Navigation Algorithm Delivery Time Hier. $=$ Hierarchical

Algorithm, Polylog. = polylogarithmic.

It is important to note that the tree creation process follows the statistical properties of the tagging dataset only; it has no inherent semantic rationale. As such, it serves primarily as a statistical tool to improve the efficiency of navigability from a network-theoretic perspective. Table 3 provides an overview of the results of the structural network analysis performed with the three real life datasets.

Another important observation is that in our model each paginated tag is a source of a network generated by a hierarchy. These networks are themselves connected through tag cooccurrence in the dataset, i.e., since tags overlap and share resources such shared resources link different generated networks. This makes it more difficult to estimate the delivery time of a decentralised search algorithm possessing only the local knowledge. If 
the algorithm is extended to have knowledge of all the hierarchies used in the generation of the networks, then this additional information might be useful in finding a destination node faster.

However, more theoretical work is needed to offer a proof of this intuitive assumption. In addition, it would be interesting to test these ideas empirically, e.g., by implementing the algorithm and applying it to the real life datasets. Another interesting problem is the fitting of parameters for the hierarchical network model, e.g., what is the optimal combination of the cluster size and the maximum number of children, with respect to the size of the resource list and the pagination parameter $k$.

\subsection{Calculation of resource hierarchies}

The hierarchy used in our experiments so far does not possess any semantic grounding. It is a synthetic hierarchy trying to optimise navigational aspects of the generated network. However, improvements of our algorithm will need to take the semantics of the dataset into account by identifying a set of resource (metadata) attributes. For example, resource attributes might be the date of creation, authors, other tags, or even attributes external to the system such as URLs, full-text, or title. Similar to tag-resource bipartite graphs, a collection of metadata attributes and resources can be always represented as yet another bipartite graph. Thus, the discussion that follows applies for arbitrary resource metadata. However, for simplicity reasons we refer henceforth only to tag-resource bipartite graphs.

Let us, here, shortly discuss possible approaches to obtain semantically useful resource hierarchies. We can calculate resource hierarchies by applying e.g., modern hierarchical clustering algorithms such as K-means (Dhillon et al., 2001) or affinity propagation (Frey and Dueck, 2007) to the tag vectors (see e.g., Plangprasopchok et al., 2010). Alternatively, if we deal with text resources it is possible to apply K-means or affinity propagation on the term vectors. However, in general case, e.g., in the case when we deal with non-textual resources such as images or videos we have only tag vectors.

In Heymann and Garcia-Molina (2006) the authors argue that similarity between tags (the tag vectors are sparse) are not sufficiently great for purely similarity based hierarchical clustering methods. Therefore, the authors designed a new algorithm tailored to the specifics of the social tagging data. This new algorithm produces so called folksonomies ${ }^{4}$ - folk-generated taxonomies - which are tag hierarchies. In Benz et al. (2010) the authors extend this idea and design yet another folksonomy creation algorithm.

The input for those folksonomy creation algorithms is the so-called tag similarity graph - an unweighted graph with tags as nodes. Two nodes are linked to each other if their similarity is above a predefined similarity threshold. In the simplest case, the threshold is defined through tag overlap - if the tags do not overlap in at least one resource than they are not linked to each other in the tag similarity graph. As the first step, the algorithm calculates node centralities producing a generality ranking where the most general tags come in the top positions. Then, the algorithm starts by a single node tree with the most general tag as the root node and proceeds by iterating through the generality ranking and adding each tag to the tree - the algorithm calculates the similarities between the current tag and each tag currently present in the tree and adds the current tag as a child to its most similar tag. 
The algorithm is extensible as it is possible to apply different similarity and centrality measures, e.g., the algorithm described in Heymann and Garcia-Molina (2006) work with the cosine similarity and closeness centrality, whereas the algorithm described in Benz et al. (2010) works with the cooccurrence and degree centrality.

The folksonomy algorithms produce tag hierarchies, however, we are interested in producing resource hierarchies. A possible approach is to adapt the folksonomy algorithms to produce global resource hierarchies or resource hierarchies of a given paginated tag instead of global tag hierarchies. Thus, the adapted algorithm:

1 maps the bipartite tag-resource graph onto a resource-resource cooccurrence graph

2 compiles a generality ranking by calculating a centrality of nodes in the resource-resource graph

3 builds the cooccurrence matrix between resources for similarity calculation

4 starts with the most general tag as the root node

5 iterates through the generality ranking and attaches the next resource from the ranking to its most similar resource from the tree.

In addition (to obtain better navigational properties), we can introduce hierarchy branching factor $b$ and add new resources to the tree only to those resources that still have available spots for child resources.

The future work can concentrate on implementation and evaluation of such algorithms. One problem that the future work needs to address is scalability resource-resource mappings of tagging datasets tend to produce huge networks with billions of links.

\section{Navigational and semantic penalty}

The previous section shows that one way of designing an efficiently navigable network in a tagging system is to classify the resources of a given paginated tag into a hierarchy. Thus, to design a navigable network, the pagination algorithm needs to organise these resource attributes into a hierarchy. At the same time, it is difficult to expect that an algorithm taking into account the semantics of resources can produce an optimal hierarchy that optimises navigability of the tagging system as a whole. Rather, the semantic algorithm will tend to produce an unbalanced tree with a variable cluster size. As a consequence, the navigational structure generated by such an algorithm will be sub-optimal, i.e., a decentralised search algorithm will need to take more steps (investigate more nodes) to find a destination node. We will call this effect the navigational penalty. Of course, the pagination algorithm might be altered to produce a tree closer to the optimal tree from the navigational point of view. This, however, seems possible only by breaking semantics to a certain extent. We will call this contrasting effect the semantic penalty. This reveals an essential trade-off which tag cloud construction algorithms will need to address: balancing the navigational and semantic penalties. 
Let us illustrate the navigational and semantic penalties with an example. Suppose we have 1,000 resources about Austrian cities tagged with 'Austria'. A particular tagging system might decide to paginate that tag with a pagination parameter of $k=20$ (listing 20 resources per page). Firstly, the system would need to semantically classify the resources into a clustered hierarchy. For example, it could take geography as the criteria for creating clusters: each cluster corresponding to an Austrian province. However, the size of the clusters varies and the province of Vienna (the capital of Austria) might dominate, since it contains, say, 500 resources. Generating the network from such an unbalanced hierarchy will result in a navigational penalty, whereas a new classification of the resources taking into account the Vienna districts as a further geographical refinement to balance the cluster size may cause a semantic penalty, if the Vienna province is represented at a finer level of detail than other provinces.

\subsection{Measuring semantic penalty}

In the following, we present a simple method for estimating the semantic penalty of different pagination algorithms.

If we ignore pagination and show the complete resource list $R_{t}$ whenever a tag $t$ is selected, $t$ is connected through this resource list to the set of its cooccurring tags. We represent the tag cooccurrence of $t$ by means of the cooccurrence vector $\mathbf{c}_{\mathbf{t}}$. The dimensions in this vector correspond to tags, and the value of a particular dimension is the number of resources that share both $t$ and the dimension tag.

Taking into account pagination, a particular selection of resources for $t$ is the set $L_{t}$, which is a function of $r, k$, and the resource hierarchy in question. We can now introduce a resource-specific cooccurrence vector of a given tag $t$ and denote it as $\mathbf{c}_{\mathbf{t}}^{\mathbf{r}}$. Again, the vector dimensions are tags, and the vector values correspond to the number of shared resources between $t$ and a particular dimension tag. However, the resources have to belong to $L_{t}$ now.

We take the complete cooccurrence vector $\mathbf{c}_{\mathbf{t}}$ of a given tag $t$ as the ground truth. The resource-specific cooccurrence vector $\mathbf{c}_{\mathbf{t}}^{\mathbf{r}}$ of $t$ is than compared against ct using cosine similarity $c s$ (cosine of the angle between vectors $\mathbf{c}_{\mathbf{t}}$ and $\mathbf{c}_{\mathbf{t}}^{\mathbf{r}}$ ) to estimate its alignment with the ground truth:

$$
c s\left(\mathbf{c}_{\mathbf{t}}, \mathbf{c}_{\mathbf{t}}^{\mathbf{r}}\right)=\frac{\mathbf{c}_{\mathbf{t}} \cdot \mathbf{c}_{\mathbf{t}}^{\mathbf{r}}}{\left\|\mathbf{c}_{\mathbf{t}}\right\|\left\|\mathbf{c}_{\mathbf{t}}^{\mathbf{r}}\right\|}
$$

In the next step, we calculate the arithmetic mean of cosine similarities over all resources of a given paginated tag $t$ :

$$
\overline{c s_{t}}=\frac{1}{\left|R_{t}\right|} \sum_{r \in R_{t}} c s\left(\mathbf{c}_{\mathbf{t}}, \mathbf{c}_{\mathbf{t}}^{\mathbf{r}}\right)
$$

Then, we calculate the arithmetic mean of $\overline{c s_{t}}$ over all tags:

$$
\overline{c s}=\frac{1}{|T|} \sum_{t \in T} \overline{c s_{t}}
$$


Finally, we obtain a single numerical value - the semantic penalty of a given pagination algorithm as:

$$
s p=1-\overline{c s}
$$

We subtract from 1 to express the fact that maximum similarity would be equivalent to the absence of any semantic penalty. In addition, we can vary the parameter $k$ to see how the semantic penalty is distributed with the size of the paginated page presented to users.

Let us illustrate the intuition behind the semantic penalty with the following example. In a given tagging dataset, semantics emerge through relations between tags, e.g., the tag Austria might be related via cooccurrence to tags such as Vienna (sharing a single resource), Europe (sharing two resources), and Alps (sharing two resources). Through pagination, some of the links disappear because resources and their corresponding tags are omitted from the resource list, e.g., after pagination Austria is related only to Vienna. Let Austria be the first dimension, Vienna the second, Europe the third, and Alps the fourth. We have:

$$
\mathbf{c}_{\text {austria }}=\left[\begin{array}{l}
0 \\
1 \\
2 \\
2
\end{array}\right] \quad \mathbf{c}_{\text {austria }}^{\mathbf{r}}=\left[\begin{array}{l}
0 \\
1 \\
0 \\
0
\end{array}\right] \quad c s=\frac{1}{3} \quad s p=\frac{2}{3}
$$

Thus, the semantic penalty measures the extent to which the list of displayed resources is semantically different from the global semantics of the tag.

Figure 4 compares the semantic penalty of the reverse chronological, random, and synthetic hierarchical (see Section 5-B) pagination algorithms over all datasets. The preliminary results show that the semantic penalty does not depend on the selection of the pagination algorithm but only on the length $k$ of the paginated list. This result is consistent over all datasets.

Although the results are only preliminary they contain an interesting observation: While the semantic penalties for smaller $k$ are still significant, as $k$ grows the semantic penalty decreases very quickly. Even though the algorithms do not optimise for semantics, paginated lists of length 20 or more do not induce significant semantic penalties. Consistently, over all datasets and all algorithms the semantic penalty for $k$ greater than 20 drops to $\approx 1 \%$. The exception here is again the Austria-Forum dataset (the semantic penalty is marginal even for small $k$ ): there are only few hub tags in the network and that reduces the pagination effect on the semantics.

Result 5 Limiting the pagination list length to practically feasible sizes (e.g., 20, 30, or more) does not introduce a significant semantic penalty.

The further investigation should evaluate semantically optimised algorithms to identify potential differences between the observed and new semantics-aware pagination algorithms. However, as the semantics is not significantly impaired by pagination (at least for higher values of $k$ ), future research can concentrate on measuring the navigational penalty and optimising pagination algorithms for navigation. 
Figure 4 The semantic penalty induced by different pagination algorithms for the three datasets, (a) Austria-Forum (b) BibSonomy (c) CiteULike (see online version for colours)

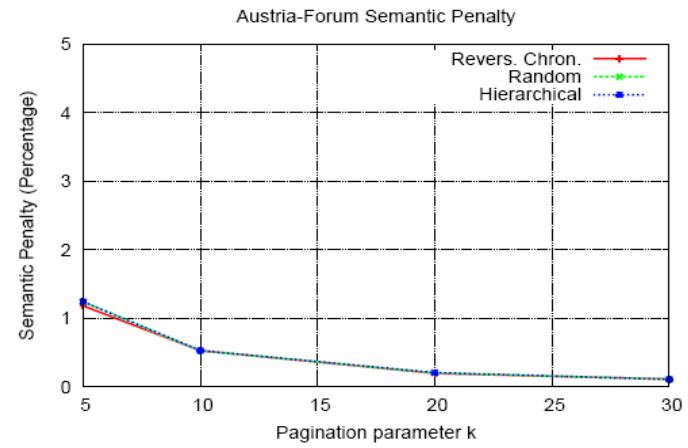

(a)

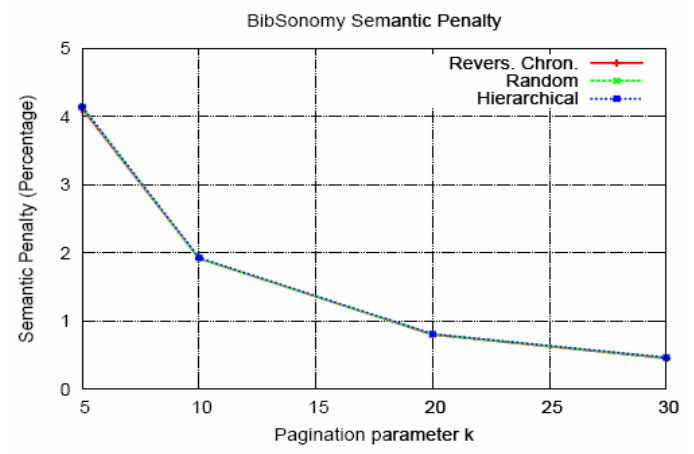

(b)

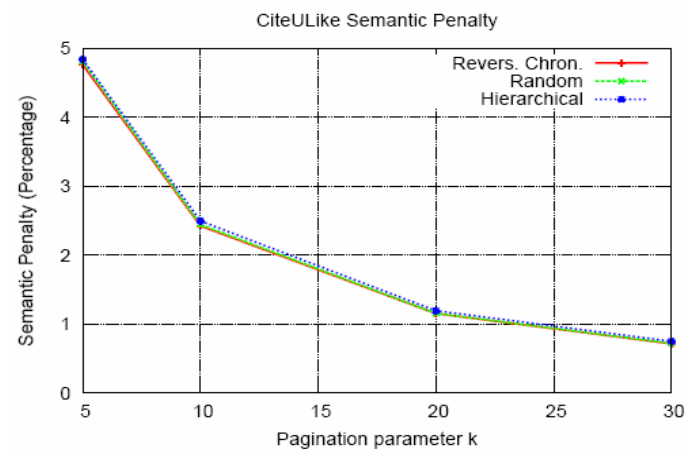

(c)

Notes: The two mature datasets (Bibsonomy and CiteULike) exhibit larger semantic penalties, while the Austria-Forum (a tagging system in an early adoption phase) exhibits significantly smaller penalties - there are fewer paginated hub tags in the Austria-Forum and therefore, the pagination effect on the semantics is marginal. The semantic penalty does not depend on the pagination algorithm but solely on the number of resources shown in the paginated list. While semantic penalty for smaller values of $k$, e.g., 5 and 10 is still significant, limiting the paginated list to a practically feasible length, e.g., 20, does not impair semantics (the semantic penalty drops to $\approx 1 \%$ ). 


\section{Related works}

We start our review of related work with a brief overview of network-related research. Research on network navigability has been inspired by Milgram's (1967) small world experiment. In this experiment, selected persons from Nebraska received a letter they were then asked to send through their social networks to a stockbroker in Boston. The striking result of the study was that, for those letters reaching the destination, the average number of hops was around six, i.e., the population of the USA constituted a 'small world'. While the conclusions have been challenged (Kleinfeld, 2002), this experiment has attracted a great deal of interest in the research community.

Numerous researchers analysed Milgram's experiment trying to create network models and generators able to produce such 'small world' networks (see e.g., Kochen, 1989). The lattice model by Watts and Strogatz (1998) mimics a real life social network, where people are primarily connected to their neighbours with a few 'long-range' contacts. The networks generated by this model have, like the random graph model (Bollobás and de la Vega, 1982), a giant component and a diameter bound by $\log N$.

Kleinberg analysed the second result of the Milgram's experiment, the ability of people to find a short path when there is such a path between two nodes (Kleinberg, $200 \mathrm{a}, 2000 \mathrm{~b}, 2001)$. He concluded that there are structural clues in such networks, which allow people to find a short path efficiently and argued that for an 'efficiently' navigable network there exists a decentralised search algorithm with delivery time polynomial in $\log N$.

Kleinberg also designed a number of network models such as 2D-grid models (Kleinberg, 2000b), hierarchical models (Kleinberg, 2001), and group models (Kleinberg, 2001), and showed that for certain combinations of parameters, efficient decentralised search algorithms exist.

Particularly, hierarchical network models (Kleinberg, 2001) are based on the idea that, in many settings, the nodes in a network might be classified according to taxonomy. The taxonomy can be represented as a $b$-ary tree and network nodes can be attached to the leaves of the tree. For each node $v$, we can create a link to all other nodes $w$ with the probability that decreases with $h(v, w)$ where $h$ is the height of the least common ancestor of $v$ and $w$ in the tree. For a constant out-degree, the nodes are clustered and then the clusters are attached to the tree. The link distribution defined by $f(h)=(h+1)^{-2} b^{-h}$ generates a navigable network with a decentralised search algorithm with delivery time of $O\left(\log _{b}^{4} N\right)$.

In related research of tagging systems, tag clouds have been characterised as a way to translate the emergent vocabulary of a folksonomy into social navigation tools (Sinclair and Cardew-Hall, 2008; Dieberger, 1997). Social navigation itself represents a multi-dimensional concept, covering a range of different issues and ideas. A distinction between direct and indirect social navigation, e.g., highlights whether navigational clues are provided by direct communication among users (e.g., via chat), or whether navigational clues are indirectly inferred from historical traces left by others (Millen and Feinberg, 2006). Based on this distinction, our work only focuses on indirect social navigation in the sense that it studies the effectiveness of traces ('tags') left by users in tagging systems. Other types of social navigation emphasise the need to show the presence of others users, to build trust among groups of users, or to encourage certain behaviour (Millen and Feinberg, 2006). 
Researchers have discussed the advantages and drawbacks of tag clouds, suggesting that tag clouds are a useful mechanism when users' search tasks are general and explorative (e.g., learn about Web 2.0), while tag clouds provide little value for specific information-seeking tasks (e.g., navigate to http://www.cnn.com) (Sinclair and Cardew-Hall, 2008). While the paper at hand focuses on network-theoretic aspects, cognitive aspects of navigation have been studied previously using, e.g., SNIF-ACT (Fu and Pirolli, 2007) and social information foraging theory (Pirolli, 2009). Other work has studied the motivations of users for tagging (Strohmaier et al., 2010; Koerner et al., 2010), and how they influence emergent semantic (as opposed to navigational) structures. The navigational utility of single tags has been investigated (Chi and Mytkowicz, 2008) with somewhat disappointing results. With time, the tags become harder and harder to use as they lose specificity and reference too many resources. Such tags are exactly those paginated tags where new pagination algorithms are needed.

Navigation models for tagging systems have been also discussed recently. In Ramezani et al. (2009), authors describe a navigation framework for tagging systems. The authors apply the framework to analyse possible attacks on tagging systems. In principle, the framework identifies a navigation channels as any combination of the basic elements of a tagging system (users, tags, and resources). Thus, the specific combination which we investigated in this paper can be summarised as the resource-tag or tag resource navigation channel.

Recent literature also discusses algorithms for the construction of tag clouds. The ELSABer algorithm ( $\mathrm{Li}$ et al., 2007) represents an example of such an effort aimed towards identifying hierarchical relationships between annotations to facilitate browsing. The work by Aouiche et al. (2008) is another example, introducing entropy-based algorithms for the construction of interesting tag clouds. However, these algorithms have not found widespread adoption in current social tagging systems. In addition, empirical studies of tagging systems have, e.g., focused on comparing navigational characteristics of tag distributions to similar distributions produced by library terms (Heymann et al., 2010).

Our work contributes to an increased theoretical understanding about the navigability of current tag cloud algorithms in social tagging systems. Our experiments identify empirical problems related to the navigability of tag clouds in three real world tagging systems.

\section{Conclusions}

The motivation for this research was to examine and test the widely held belief that tag clouds support efficient navigation in social tagging systems. We have shown that for certain specific, but popular, tag cloud scenarios, the so-called navigability assumption does not hold. The results presented in this paper make a theoretical and an empirical argument against existing approaches to tag cloud construction. Our work thereby both confirms and refutes the assumption that current tag cloud incarnations are a useful tool for navigating social tagging systems. While we confirm that tag-resource networks have efficient navigational properties in theory, we show that popular user interface decisions (such as 'pagination' combined with reverse-chronological listing of resources) significantly impair navigability. Our experimental results demonstrate that popular 
approaches to using tag clouds for navigational purposes suffer from significant problems.

Building on recent research results from network theory, in particular hierarchical network models, we have illustrated a path towards constructing more efficiently navigable tag cloud networks, which are less vulnerable to pagination influences. Our findings suggest that engineers who want to design effective tag cloud algorithms have to essentially strike a balance between semantic and navigation penalties, in order to make navigation in social tagging systems both efficient and effective. We also presented a simple method for estimating the semantic penalty. The method is based on measuring cosine similarity between the non-paginated (ground truth) and algorithmically generated paginated tag cooccurrence vectors. The future work needs to investigate the possibilities for measuring the navigational penalty.

We conclude that in order to make full use of the potential of tag clouds for navigating social tagging systems, new and more sophisticated ways of thinking about designing tag cloud algorithms are needed.

\section{References}

Adamic, L.A., Lukose, R.M., Puniyani, A.R. and Huberman, B.A. (2001) 'Search in power-law networks', Physical Review E, September, Vol. 64, No. 4, pp.046 135 1-8.

Aouiche, K., Lemire, D. and Godin, R. (2008) 'Web 2.0 OLAP: from data cubes to tag clouds', 4th International Conference, WEBIST 2008, Vol. 18, available at http://www.springerlink.com/index/10.1007/978-3-642-01344-7.

Benz, D., Hotho, A. and Stumme, G. (2010) 'Semantics made by you and me: self-emerging ontologies can capture the diversity of shared knowledge', in Proc. of the 2nd Web Science Conference (WebSci10), Raleigh, NC, USA.

Bollobás, B. and Chung, F.R.K. (1988) 'The diameter of a cycle plus a random matching', SIAM J. Discret. Math., Vol. 1, No. 3, pp.328-333.

Bollobás, B. and de la Vega, W.F. (1982) 'The diameter of random regular graphs', Combinatorica, Vol. 2, No. 2, pp.125-134.

Cattuto, C., Schmitz, C., Baldassarri, A., Servedio, V.D.P., Loreto, V., Hotho, A., Grahl, M. and Stumme, G. (2007) 'Network properties of folksonomies', AI Commun., Vol. 20, No. 4, pp.245-262.

Chi, E.H. and Mytkowicz, T. (2008) 'Understanding the efficiency of social tagging systems using information theory', in HT '08: Proceedings of the Nineteenth ACM Conference on Hypertext and Hypermedia, pp.81-88, ACM, New York, NY, USA.

Dhillon, I., Fan, J. and Guan, Y. (2001) 'Efficient clustering of very large document collections', in Grossman, R., Kamath, C. and Naburu, R. (Eds.): Data Mining for Scientific and Engineering Applications, Kluwer Academic Publishers.

Dieberger, A. (1997) 'Supporting social navigation on the world wide web', Int. J. Hum.-Comput. Stud., Vol. 46, No. 6, pp.805-825.

Eda, T., Uchiyama, T., Uchiyama, T. and Yoshikawa, M. (2009) 'Signaling emotion in tag clouds', in $W W W$ '09: Proceedings of the 18th International Conference on World Wide Web, pp.1199-1200, ACM, New York, NY, USA.

Erdos, P. and Renyi, A. (1960) 'On the evolution of random graphs', Publ. Math. Inst. Hung. Acad. Sci., Vol. 5, pp.17-61.

Frey, B.J.J. and Dueck, D. (2007) 'Clustering by passing messages between data points', Science, January, available at http://dx.doi.org/10.1126/science.1136800. 
Fu, W-T. and Pirolli, P. (2007) 'Snif-act: a cognitive model of user navigation on the World Wide Web', Hum.-Comput. Interact., Vol. 22, No. 4, pp.355-412.

Hearst, M.A. and Rosner, D. (2008) 'Tag clouds: data analysis tool or social signaller?', in HICSS '08: Proceedings of the Proceedings of the 41st Annual Hawaii International Conference on System Sciences, IEEE Computer Society, Washington, DC, USA.

Heymann, P. and Garcia-Molina, H. (2006) 'Collaborative creation of communal hierarchical taxonomies in social tagging systems', Stanford InfoLab, Technical Report 2006-10, April, available at http://ilpubs.stanford.edu:8090/775/.

Heymann, P., Paepcke, A. and Garcia-Molina, H. (2010) 'Tagging human knowledge', in Proceedings of the Third ACM International Conference on Web Search and Data Mining, pp.51-61, ACM, New York, NY, USA.

Hotho, A., Jäschke, R., Schmitz, C. and Stumme, G. (2006) 'Bibsonomy: a social bookmark and publication sharing system', in Proceedings of the Conceptual Structures Tool Interoperability Workshop at the 14th International Conference on Conceptual Structures, pp.87-102.

Kaser, O. and Lemire, D. (2007) 'Tag-cloud drawing: algorithms for cloud visualization', Proceedings of Tagging and Metadata for Social Information Organization (WWW 2007), available at http://arxiv.org/abs/cs/0703109v2.

Kleinberg, J.M. (2000a) 'Navigation in a small world', Nature, August, Vol. 406, No. 6798.

Kleinberg, J.M. (2000b) 'The small-world phenomenon: an algorithmic perspective', in Proceedings of the 32nd ACM Symposium on Theory of Computing.

Kleinberg, J.M. (2001) 'Small-world phenomena and the dynamics of information', in Advances in Neural Information Processing Systems (NIPS), MIT Press, Vol. 14, p.2001.

Kleinfeld, J. (2002) 'Could it be a big world after all? The six degrees of separation myth', Society, April.

Kochen, M. (Ed.) (1989) The Small World, Ablex, Norwood, NJ.

Koerner, C., Kern, R., Grahsl, H.P. and Strohmaier, M. (2010) 'Of categorizers and describers: an evaluation of quantitative measures for tagging motivation', in 21st ACM SIGWEB Conference on Hypertext and Hypermedia (HT 2010), June, Toronto, Canada, ACM, Toronto, Canada.

Leskovec, J., Kleinberg, J. and Faloutsos, C. (2005) 'Graphs over time: densification laws, shrinking diameters and possible explanations', in KDD '05: Proceedings of the Eleventh ACM SIGKDD International Conference on Knowledge Discovery in Data Mining, pp.177-187, ACM, New York, NY, USA.

Li, R., Bao, S., Yu, Y., Fei, B. and Su, Z. (2007) 'Towards effective browsing of large scale social annotations', Proceedings of the 16th International Conference on World Wide Web, p.952, available at $\mathrm{http}: / /$ portal.acm.org/citation.cfm?id=1242700.

Mesnage, C.S. and Carman, M.J. (2009) 'Tag navigation', in SoSEA '09: Proceedings of the 2nd International Workshop on Social Software Engineering and Applications, pp.29-32, ACM, New York, NY, USA.

Mika, P. (2007) 'Ontologies are us: a unified model of social networks and semantics', Web Semantics: Science, Services and Agents on the World Wide Web, Vol. 5, No. 1, pp.5-15.

Milgram, S. (1967) 'The small world problem', Psychology Today, Vol. 1, pp.60-67.

Millen, D. and Feinberg, J. (2006) 'Using social tagging to improve social navigation', in Workshop on the Social Navigation and Community Based Adaptation Technologies, Citeseer, Dublin, Ireland, available at http://citeseerx.ist.psu.edu/viewdoc/download?doi=10.1.1.92.5563\&rep=rep1\&type=pdf.

Neubauer, N. and Obermayer, K. (2009) 'Hyperincident connected components of tagging networks', in HT '09: Proceedings of the 20th ACM Conference on Hypertext and Hypermedia, pp.229-238, ACM, New York, NY, USA.

Pirolli, P. (2009) 'An elementary social information foraging model', in Proceedings of the 27th International Conference on Human Factors in Computing Systems, ACM, pp.605-614. 
Plangprasopchok, A., Lerman, K. and Getoor, L. (2010) 'Growing a tree in the forest: constructing folksonomies by integrating structured metadata', in Proc. of the International Conference on Knowledge Discovery and Data Mining (KDD), July.

Ramezani, M., Sandvig, J., Schimoler, T., Gemmell, J., Mobasher, B. and Burke, R. (2009) 'Evaluating the impact of attacks in collaborative tagging environments', in International Conference on Computational Science and Engineering, 2009. CSE '09, August, Vol. 4, pp.136-143.

Rivadeneira, A.W., Gruen, D.M., Muller, M.J. and Millen, D.R. (2007) 'Getting our head in the clouds: toward evaluation studies of tag clouds', in CHI '07: Proceedings of the SIGCHI Conference on Human Factors in Computing Systems, pp.995-998, ACM, New York, NY, USA.

Schmitz, C., Hotho, A., Jäschke, R. and Stumme, G. (2006) 'Mining association rules in folksonomies', in Data Science and Classification: Proc. of the 10th IFCS Conf., Studies in Classification, Data Analysis, and Knowledge Organization, Springer, pp.261-270.

Sinclair, J. and Cardew-Hall, M. (2008) 'The folksonomy tag cloud: when is it useful?', Journal of Information Science, Vol. 34, p.15, available at http://jis.sagepub.com/cgi/content/abstract/34/1/15.

Strohmaier, M., Koerner, C. and Kern, R. (2010) 'Why do users tag? Detecting users' motivation for tagging in social tagging systems', in International AAAI Conference on Weblogs and Social Media (ICWSM2010), 23-26 May, Washington, DC, USA.

Watts, D.J. and Strogatz, S.H. (1998) 'Collective dynamics of small-world networks', Nature, June, Vol. 393, No. 6684, pp.440-442.

Watts, D.J., Dodds, P.S. and Newman, M.E.J. (2002) 'Identity and search in social networks', Science, Vol. 296, pp.1302-1305.

Zhang, Y., Jansen, B. and Spink, A. (2009) 'Time series analysis of a web search engine transaction log', Information Processing \& Management, Vol. 45, No. 2, pp.230-245.

\section{Notes}

1 Available at http://www.austria-lexikon.at.

2 Available at http://www.kde.cs.uni-kassel.de/ws/dc09/.

3 Available at http://www.citeulike.org/faq/data.adp.

4 Available at http://www.vanderwal.net/folksonomy.html. 